\title{
Intoxicación por plomo secundaria a alojamiento de esquirlas en el cuerpo.
}

\section{Lead poisoning secondary to retained bullet fragments}

\author{
Vivian Charris, Agustín Guerrero, Carlos Barrera \\ - Barranquilla (Colombia)
}

\section{Resumen}

La intoxicación por plomo es una entidad difícil de diagnosticar en sus estadios iniciales, por presentar síntomas inespecíficos que suelen ser atribuidos a otras patologías. Siendo así de gran importancia contar con una completa historia clínica que nos conduzca a la determinación de probables medios de exposición a este metal, el cual continúa siendo de alto uso en nuestro medio. No olvidar sus principales blancos de toxicidad como son el riñón, sistema nervioso y hematológico; logrando su confirmación diagnóstica con niveles elevados de plombemia y zinc protoporfirina. El tratamiento consiste en alejar al paciente de la fuente de exposición y tratamiento quelante. (Acta Med Colomb 2011; 36: 200-203)

Palabras clave: plomo, saturnismo.
Abstract
Lead poisoning is difficult to diagnose in its early stages because its symptoms are nonspecific and are often attributed to other diseases. It is therefore very important to take a complete medical history that can lead to determine probable means of exposure to this metal, which continues to be widely used in our environment. It is crucial not to forget the main targets of lead toxicity, such as the kidney, the nervous system, and the blood. Diagnosis is confirmed by elevated blood lead levels and elevated zinc protoporphyrin levels. Treatment involves withdrawing the patient from the source of exposure and chelation therapy. (Acta Med Colomb 2011; 36: 200-203)
Key words: lead, saturnism.

Dra. Vivian Charris Roldán: Residente II
Medicina Interna, Universidad Libre; Dr.
Agustín Guerrero Salcedo: Toxicólogo
Salubrista Ocupacional, Magíster en
Toxicología, Profesor Titular-Coordinador
de Farmacología y Toxicología, Facultad
de Medicina Universidades Libre y San
Martín de Barranquilla; Dr. Carlos Barrera
Guarín: Jefe Medicina Interna, Director
Postgrado Medicina Interna, Unilibre. Hos-
pital Universitario CARI Alta Complejidad.
Barranquilla (Colombia).
Correspondencia: Dra Vivian Charris.
Barranquilla (Colombia).
E-mail: viviancharris@hotmail.com
Recibido: 12/VI/201 aceptado: 06/X/2011

\section{Introducción}

El plomo es obtenido por fundición o refinamiento de minas o reciclamiento de materiales de desecho que lo contengan (1). Las principales exposiciones proceden del ambiente laboral en el sector de la metalurgia y minería extractiva, así como en la industria informal. La ingesta de alimentos contaminados, cocinados o guardados en utensilios de alfarería glaseada o cristal emplomado y bebidas alcohólicas de fabricación clandestina destiladas o depositadas en recipientes de este metal. La absorción percutánea del plomo inorgánico es mínima, pero el orgánico se absorbe bien por esta. Intoxicaciones contemporáneas en países desarrollados pueden ser atribuidas a distribución de alimentos en envases o envoltorios que contienen plomo, también en las drogas ilícitas contaminadas y el plomo liberado de proyectiles o esquirlas alojadas en el cuerpo (2).
Tabla 1. Paraclínicos al ingreso del paciente.

\begin{tabular}{|l|c|}
\hline Paraclínicos de ingreso & Valores \\
\hline Hemoglobina & $7.2 \mathrm{gr}$ \\
\hline Hematocrito & $21.5 \%$ \\
\hline VCM & $73.6 \mathrm{fl}$ \\
\hline HCM & $24 \mathrm{fl}$ \\
\hline BUN & 20.9 \\
\hline Creatinina & $1.28 \mathrm{mg}$ \\
\hline Urea & 44.7 \\
\hline Plomo sérico & $76.30 \mathrm{ug} / \mathrm{dl}$ \\
\hline Plomo en orina de 24 hrs & $0.064 \mathrm{mg} / \mathrm{l}$. \\
& Normal: hasta $0.06 \mathrm{mg} / \mathrm{dl}$ \\
\hline Ácido Delta Amino Levulínico & $146.1 \mathrm{mg} / 24 \mathrm{hrs}$. \\
en orina de 24 hrs & Normal:1.3-7.0 mg/24hrs \\
\hline
\end{tabular}


Los primeros datos históricos acerca del plomo liberado por proyectiles, se remonta a 1911 cuando Lewin, cirujano alemán, hizo un recuento de diferentes casos clínicos enumerando los posibles mecanismos fisiopatológicos en la génesis de la intoxicación plúmbica secundaria a presencia de balas intraarticulares (3), entre ellos señala la alcalinidad del líquido sinovial y alto contenido lipoide, el cual disuelven el plomo y hacen que se deposite en tejido subsinovial, ya que aquí se encuentra en forma libre, no ligada a proteínas, causando irritación local y pasando luego al torrente sanguíneo a través del sistema linfático regional. Se logró determinar que para producir saturnismo se requieren más de $40 \mathrm{gm}$ de esquirlas plomadas en tejido celular subcutáneo y músculo (4). Se conoce que algunos estados hipermetabólicos como estrés, infecciones, endocrinopatías o alcoholismo pueden ser factores desencadenantes para el desarrollo del saturnismo en un paciente con balas retenidas (5).

\section{Presentación del caso}

Masculino, 19 años de edad, cuadro clínico de tres años de evolución, posterior a recibir herida por escopeta, en cadera izquierda, sin lesión ósea, vascular o nerviosa conocida, sin extracción de esquirlas. Un año después, inicia cuadro de epigastralgia, naúseas, vómitos, pérdida progresiva de peso y anemia, manejado como parasitosis intestinal. Posteriormente astenia, adinamia, disminución progresiva y ascendente de fuerza muscular en cuatro extremidades, anemia persistente, disminución de agudeza visual, constipación, vértigo y parestesias. Al examen físico extremidades atróficas, con flexión permanente de dedos de la mano y paresia en cuatro extremidades (Tabla 1).

TAC de cadera simple: fractura base-cervical izquierda desplazada. Marcada disminución en amplitud del espacio articular coxofemoral, esclerosis cortical, numerosos fragmentos con densidad metálica $(20 \mathrm{~mm}$ en compartimiento anterior, $30 \mathrm{~mm}$ adyacente al trocante mayor y otros inferiores a este ascendente a la cortical media del tercio proximal de la diáfisis femoral de $45 \mathrm{~mm}$ el de diámetro mayor, otras esquirlas múltiples no superan $5 \mathrm{~mm}$ ). Diagnosticándose así, intoxicación crónica por plomo, con compromiso hematológico, renal y neuropático. Es intervenido por ortopedia realizándose extracción de esquirlas y reemplazo de cadera. Se inicia manejo con penicilamina diaria por tres semanas, con los siguientes controles paraclínicos a su egreso: plomo sérico: $31.2 \mathrm{ug} / \mathrm{dL}$, plomo en orina de $24 \mathrm{hrs:} 0.010 \mathrm{mg} / \mathrm{dL}$. creatinina: $0.85 \mathrm{mg}$ y hemoglobina: $10 \mathrm{gr}$ egresando a casa con resolución de síntomas y secuelas de polineuropatía periférica confirmada por electromiografía.

\section{Discusión}

La absorción y distribución del plomo depende de forma, tamaño, tránsito gastrointestinal, estado nutricional y edad; hay mayor absorción ante partículas pequeñas, deficiencia de hierro y/o calcio, gran ingesta de grasa, inadecuada ingesta de calorías, ayuno y niñez, ya que en ellos la absorción es de $30-50 \%$ mientras en adultos es $10 \%$ (1). Inicialmente circula en sangre, unido el $95 \%$ al eritrocito, el mecanismo no está bien dilucidado, al parecer, el primer lugar de fijación es la membrana celular. La sangre transporta el plomo hacia todo el cuerpo y lo deposita en tejidos de acuerdo con un gradiente de concentración y afinidad específica de cada órgano. Luego se distribuye a tejidos como hígado, riñón, médula ósea y sistema nervioso central que son los órganos blancos de toxicidad, luego de uno a dos meses difunde a huesos donde es inerte y no tóxico; aproximadamente $90 \%$ de carga corporal de plomo se une firmemente a este tejido. La concentración máxima de plomo en huesos se alcanza hacia quinta o sexta década, para luego disminuir (2). Este metal puede movilizarse del hueso en situaciones como inmovilidad, embarazo, hipertiroidismo, medicaciones y edad avanzada. Además puede cruzar placenta y barrera hematoencefálica (1).

La excreción del plomo es casi toda por vía renal y se hace por filtración glomerular, excretándose por orina en un $90 \%$, y en menor cantidad en bilis, piel, cabello, uñas, sudor y leche materna. No se conoce exactamente la vida biológica media del plomo, pero se acepta variaciones amplias entre 15 y 27 años en adultos (2).

El plomo es tóxico para enzimas dependientes del zinc. Interfiere con síntesis del Hem, ya que se une a grupos sulfhidrilos de enzimas como la d-aminolevulínico deshidratasa, coproporfirinógeno oxidasa y ferroquelatasa, siendo el resultado final aumento de protoprofirinas como zinc-protoporfirina (ZPP) y anemia (1).

El mecanismo de acción del plomo es complejo; parece ser que el plomo interfiere con el metabolismo del calcio, sobre todo cuando el metal está en concentraciones bajas, alterándolo de las siguientes formas: a) reemplaza al calcio y se comporta como un segundo mensajero intracelular, alterando la distribución del calcio en compartimientos intracelulares; b) activa la proteinquinasa $\mathrm{C}$, enzima que depende del calcio y que interviene en múltiples procesos intracelulares; c) se une a la calmodulina más ávidamente que el calcio; d) inhibe la bomba Na-K-ATPasa, aumentando el calcio intracelular. Finalmente estas alteraciones traerían consecuencias en neurotransmisión y tono vascular, lo que explicaría en parte la hipertensión y neurotoxicidad. A nivel renal interfiere con la conversión de vitamina $\mathrm{D}$ a su forma activa, hay inclusiones intranucleares en los túbulos renales, produce tubulopatía, que en estadios más avanzados llega a atrofia tubular y fibrosis sin compromiso glomerular, caracterizándose por proteinuria selectiva $(2,6)$. El plomo se acumula en el espacio endoneural de nervios periféricos causando edema, aumento de presión en dicho espacio y finalmente daño axonal (6). En el hueso se realiza la medición más significativa de exposición acumulada al plomo (2).

El cuadro clínico puede ser de tipo agudo o crónico. y las manifestaciones características de cada una se pueden ver en la Tabla 2. Existe correlación entre los niveles séricos de plomo y el cuadro clínico, como se observa en la Tabla 3. 
Tabla 2. Manifestaciones características de las intoxicaciones agudas y crónicas por plomo.

\begin{tabular}{|l|l|}
\hline Toxicidad aguda & $\begin{array}{l}\text { Exposición respiratoria. } \\
\text { Manifestaciones: insuficiencia renal, síntomas gastrointesti- } \\
\text { nales y encefalopatía. Predomina hemolisis y anemia. } \\
\text { Puede desarrollar en semanas e incluso días (2). }\end{array}$ \\
\hline Toxicidad crónica & $\begin{array}{l}\text { Más frecuente. } \\
\text { Manifestación con compromiso multisistémico. } \\
\text { La primera fase: intoxicación "leve" o "subclínica". } \\
\text { Saturnismo o intoxicación clínica, para fases más severas (2). }\end{array}$ \\
\hline
\end{tabular}

A menudo los síntomas comienzan en forma insidiosa y pueden pasar desapercibidos. Se inicia con síntomas vagos como astenia, adinamia, insomnio, acompañado de alteración bioquímica. Progresivamente, hay cambios en humor, irritabilidad y fatiga fácil. Al continuar la absorción o si aumenta bruscamente, aparecen síntomas más definidos, como insomnio severo, cefalea, sabor dulce y metálico (2). En esta fase, predominan síntomas gastrointestinales, incluyendo pérdida de apetito, epigastralgia, meteorismo, constipación o diarrea. Además mialgias, hipersensibilidad en articulaciones y entumecimiento de miembros inferiores.

$\mathrm{Al}$ examen clínico se puede encontrar:

- Temblor fino e hiperreflexia con neuropatía periférica leve detectable con electromiografía $(2,6)$.

- Polineuropatía periférica. Los expuestos crónicamente, sin medidas de protección pueden presentarla, afecta predominantemente miembros superiores, más los músculos extensores que flexores y más el lado dominante, lo que se llama "mano del pintor" $(2,6)$. En este momento hay niveles de AAL y CPU elevadas: $40-50 \mathrm{mg} / \mathrm{L}$ y $1000-$ $2000 \mathrm{ug} / \mathrm{L}$, respectivamente $(2,8)$.

- "Cólico saturnínico" es el siguiente paso en la clínica si no se toma acción correctiva. se caracteriza por ataques de dolor con defensa abdominal, el dolor puede ceder con la presión del abdomen $(2,1)$.

- Ribete de Burton" o línea de sulfuro. Algunos pacientes con mala higiene oral pueden presentarlo, consiste en una línea oscura entre la base del diente y encía, debido a que el sulfuro liberado por las bacterias se une al plomo (sulfuro de plomo) (1). En esta fase, los parámetros hematológicos están francamente alterados. La hemoglobina, a nivel del mar, cae cerca de 8 gr, pero en la altura raramente baja menos de 13. Sin embargo, el AAL urinario suele ser mayor de $100 \mathrm{mg} / \mathrm{L}$ y CPU está entre 5000 y $7000 \mathrm{ug} / \mathrm{L}$, la zinc protoporfirina o ferroporfirina eritrocitaria (ZPP/FEP) excede los $500 \mathrm{ug} / 100 \mathrm{~mL}$. En esta fase, el plomo puede alcanzar los $100 \mathrm{ug} / 100 \mathrm{~mL}$, sobre todo si hay cólico persistente o recurrente. Aquí, el riesgo de paresia de nervios periféricos, nefropatía y encefalopatía es mayor (2).

- Encefalopatía plúmbica, caracterizada por trastorno del sensorio y convulsiones, se presenta con $\mathrm{Pb}-\mathrm{S}$ mayor de $100 \mathrm{mg} / \mathrm{dL}$ (2) (Tabla 3).

En los exámenes de laboratorio es posible hallar:
Tabla 3. Correlación entre el plomo sérico y el cuadro clínico.

\begin{tabular}{|c|c|}
\hline Plomo sérico ug/dL & Manifestaciones clínicas \\
\hline$<10$ & Inhibición dehidratasa del ácido aminolevulínico. \\
\hline $15-29$ & $\begin{array}{l}\text { Elevación de protoporfirina eritrocitaria, aumento de ex- } \\
\text { creción urinaria de ácido aminolevulínico y coproporfirina } \\
\text { urinaria (CPU). }\end{array}$ \\
\hline $30-39$ & Hipertensión leve, alteración función testicular. \\
\hline $40-49$ & Disminución de conducción nerviosa periférica. \\
\hline $50-59$ & $\begin{array}{l}\text { Anemia. } \\
\text { Cólico saturnínico. } \\
\text { Síntomas neurológicos. }\end{array}$ \\
\hline $60-69$ & Alteración reproductiva femenina. \\
\hline $70-90$ & $\begin{array}{l}\text { Anemia severa. } \\
\text { Nefropatía. } \\
\text { Síntomas de encefalopatía. }\end{array}$ \\
\hline$>100$ & $\begin{array}{l}\text { Signos de encefalopatía. } \\
\text { Nefropatía crónica }(1,2,6-8) \text {. }\end{array}$ \\
\hline
\end{tabular}

- Anemia que puede ser normocrómica e hipocrómica (6).

- Punteado basófilo no es patognomónico, pero sí característico del saturnismo (6).

- B2 microglobulina en orina: marcador temprano de daño renal (6).

- Espermatograma: puede hallarse alteración tanto en número como forma de espermatozoides (6).

- Plombemia: indica exposición y sirve para tomar conducta terapéutica (6).

- Zinc-protoporfirina: marcador de efecto, indica daño de órgano blanco, en este caso hematopoyético $(1,7,8)$.

El tratamiento se basa en: alejamiento de la fuente de exposición y tratamiento quelante (Tabla 4), si la plombemia es $>60 \mathrm{ug} / \mathrm{dL}$ o según la clínica $(2,6)$.

\section{Conclusiones}

La documentación de este caso clínico nos permite evidenciar cómo una clínica inespecífica y un diagnóstico poco pensado nos lleva a pasar por alto este tipo de intoxicación por metales. Evidenciamos cómo en esta situación, un pa-

Tabla 4. Quelantes útiles en la intoxicación por plomo.

\begin{tabular}{|l|l|}
\hline Quelantes & \multicolumn{1}{|c|}{ Uso } \\
\hline Edetato-disódico-cálcico(EDTA Ca) & $\begin{array}{l}\text { Asociado con precipitar la encefalopatía } \\
\text { plúmbica en pacientes con absorción continua } \\
\text { de plomo y con alta carga corporal de este } \\
\text { metal; por lo que en casos de encefalopatía o } \\
\text { plombemia mayor a 100 } \mathrm{mg} / \mathrm{dl} \text { se debe asociar } \\
\text { a dimercaprol(BAL) }(2,6) .\end{array}$ \\
\hline Ácido dimercaptosuccínico(DMSA) & $\begin{array}{l}\text { Ventaja que provoca pocos efectos adversos y } \\
\text { se usa vía oral. Este quelante no redistribuye } \\
\text { el plomo a cerebro }(2,6) .\end{array}$ \\
\hline Penicilamina & $\begin{array}{l}\text { Como alternativa en nuestro medio en ausencia } \\
\text { de EDTA o DMSA puede suministrarse. } \\
\text { Actúa aumentando la excreción urinaria de } \\
\text { plomo, pero no tan específicamente como el } \\
\text { Edetato. No se conoce su mecanismo, ni lugar } \\
\text { específico de acción }(2,6) .\end{array}$ \\
\hline
\end{tabular}


ciente joven, multiconsultante, con sintomatología inicialmente inespecífica, fue manejado de forma refractaria como parasitosis intestinal. Al correlacionar datos clínicos como anemia refractaria a manejo, daño renal y polinerupatía, se pudo llegar a la sospecha clínica de que su antecedente de herida por arma de fuego y el depósito actual de esquirlas adyacentes a una articulación pudieran estar correlacionadas con sus manifestaciones clínicas; encontrándose así niveles de plomo sérico elevados. Debido a la ausencia de los quelantes de primera línea recurrimos a la penicilamina y al manejo conjunto por ortopedia para alejar el factor causal. Con todo esto logramos normalización de los niveles de plombemia, corrección de la anemia y de la falla renal, pero desafortunadamente persiste como secuela la neuropatía periférica secundaria a su intoxicación.

Es de vital importancia hacer seguimiento a pacientes con proyectiles o esquirlas retenidas, aun más si éstas se encuentran en áreas articulares. Realizando determinaciones periódicas de plomo sérico y vigilando la presencia de signos de saturnismo. Ya que el diagnóstico de esta entidad suele ser difícil, debido a que el cuadro clínico es sutil y los síntomas inespecíficos.
Ante hallazgos de aumento de niveles de plomo sérico o datos de saturnismo, hay que extraer el proyectil o esquirlas, e iniciar manejo médico para evitar complicaciones.

A nivel mundial hay registro de casos de intoxicación por plomo secundario a proyectiles alojados en el cuerpo. En nuestra ciudad no existen publicaciones al respecto. Se presenta éste por encontrarse bien documentado y por el interés académico, sociocultural y científico que representa.

\section{Referencias}

1. Melinda M. Valdivia Infantas. Intoxicación por plomo. Rev Soc Per Med Inter 2005; 18(1): 22-7.

2. Ramírez A. El cuadro clínico de la intoxicación ocupacional por plomo. Universidad Nacional Mayor de San Marcos. An Fac Med Lima 2005; 66.

3. Lewin L. Das toxische Verhalten von metallischen Blei und besonders von Blugeschossen in tierischen Korpern. Archiv Klln Chir Berl 1991; 94: 937-72.

4. Bargagna M. In tema di valutazione medico-legale della intossicazione da piombo e retenzione di proiettili. G Med Legale Infort TosslcoI 1960; 6:1.

5. Linden MA. Lead poisoning from retained bullets. Ann Surg 1982; 195: 305-13.

6. McGuigan M. Chronic poisoning: trace metals and others, Goldman: Cecil Medicine, 23rd ed. 2007

7. Ferri F. Lead poisoning, Ferri's Clinical Advisor 2010; 1st ed.

8. Shannon. Lead poisoning in adults, Haddad and Winchester's Clinical Management of Poisoning and Drug Overdose, 4th ed. 2007. 